\title{
Small bowel malignancy in patients undergoing capsule endos- copy at a tertiary care academic center: Case series and review of the literature
}

다(1) $(9)$

\author{
Authors \\ Institutions \\ 1 College of Medicine and Veterinary Medicine, \\ The University of Edinburgh, Edinburgh, UK \\ 2 Centre for Liver and Digestive Disorders, The Royal \\ Infirmary of Edinburgh, Edinburgh, UK \\ 3 Ninewells Hospital and Medical School, Dundee, UK
}

Connor A Johnston ${ }^{1}$, Diana E Yung ${ }^{2}$, Alka Joshi ${ }^{3}$, John N Plevris ${ }^{1,2}$, Anastasios Koulaouzidis ${ }^{2}$ at a Scottish tertiary center. submitted 9.9.2016

accepted after revision 2.3.2017

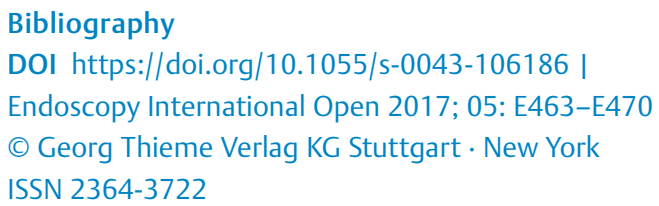

Corresponding author

Dr Diana E Yung, Centre for Liver and Digestive Disorders, The Royal Infirmary of Edinburgh, 51 Little France Crescent, Edinburgh EH16 4SA

diana.e.yung@gmail.com

\section{ABSTRACT}

Background and study aims Small bowel cancer is rare, accounting for $<5 \%$ of all gastrointestinal neoplasms. Capsule endoscopy has become the procedure of choice for non-invasive diagnosis of small bowel diseases. Data on capsule endoscopy diagnosis of small bowel cancer are limited. The objective of the study was to determine the frequency, indications and diagnostic work-up of patients with small bowel malignancy found by capsule endoscopy

Patients and methods In this retrospective study, records all patients who underwent small bowel capsule endoscopy at our center over a 10 -year period were reviewed for possible malignancy. Further data were gathered on preceding and subsequent investigations, management and outcome of these patients.

Results From 1949 studies, small bowel malignancies were diagnosed in only 7 patients $(0.36 \%$; $2 \mathrm{~F} / 5 \mathrm{M}$; median age 50 , range $34-67)$. The main indication was iron-deficiency anemia $(n=5)$. Prior to capsule endoscopy, 6 of 7 patients had bidirectional endoscopies and one had gastroscopy. All prior investigations were normal or nondiagnostic. Two of 7 experienced capsule retention. Five of 7 underwent surgery. Four patients died, giving a 5-year survival rate of $42.9 \%$.

Conclusion Small bowel malignancies diagnosed by capsule endoscopy are rare, and the median age of 50 indicates they are more common in relatively younger patients. Capsule endoscopy is effective at diagnosing a rare malignancy when other imaging modalities have failed.

\section{Introduction}

Tumors of the small bowel are rare. The small bowel accounts for $75 \%$ of the length and over $90 \%$ of the mucosal surface of the gastrointestinal tract; however, small bowel cancers represent only $1 \%$ to $3 \%$ of all primary gastrointestinal malignancies [1-3]. The clinical presentation of small bowel cancers is nonspecific with diarrhoea, abdominal pain, bleeding, extraintestinal symptoms (such as paraneoplastic phenomena) or acute small bowel obstruction [4]. Diagnosis of small bowel tumors is often delayed $[5,6]$, partly due to the fact that they are usually asymptomatic at the early stage [7] and there is low clinical suspicion. By the time a diagnosis is reached, approximately $50 \%$ of small bowel cancers have already metastasised [8].
Nowadays, capsule endoscopy (CE) is a widely-accessible and minimally invasive mode for diagnostic investigation of the small bowel [9]. CE also gives detailed and panoramic views of the entire small bowel, therefore it is suitable as first line in the diagnostic workup of small bowel tumors. Since its introduction into clinical practice, the literature on small bowel cancer diagnosis by CE remains limited [10]. This study aims to describe the frequency, indications and diagnostic work-up of patients with small bowel malignancy found by CE at a tertiary referral center in Scotland for small bowel capsule endoscopy (SBCE). 


\section{Patients and methods}

This retrospective study involved all patients who underwent SBCE between March 2005 and October 2015 at the Centre for Liver and Digestive Disorders, Royal Infirmary of Edinburgh, Scotland. Patients excluded from CE were those with known small bowel obstruction, implanted cardiac pacemakers and/ or pregnancy. Patients with risk factors for capsule retention, such as those with a high probability of small bowel stenotic lesion or known small bowel inflammation (e.g. Crohn's disease) underwent CE provided there were no obstructive symptoms. The standard protocol for performing CE involved a 12-hour fast before the procedure, with intake of 2 liters of bowel preparation solution (polyethylene glycol (PEG) before 2013, and sodium picosulphate from 2013). Patients underwent CE using one of two capsule models; either PillCam ${ }^{\mathrm{TM}}$ SB (Medtronic, USA) or MiroCam ${ }^{\mathrm{TM}}$ (IntroMedic, Seoul, Korea). At our center, all CEs are reported by at least 1 of 3 experienced CE readers (experience with $>100$ CEs before the start of this study).

The data were collected from a prospectively-designed database of all patients undergoing SBCE at our center. Patient notes from the centralized patient management platform for our healthcare trust were searched to determine those in whom a small bowel malignancy was confirmed. Further data were then gathered on previous and subsequent diagnostic investigations, management and outcome for these patients.

Data gathered: age, gender, indication for SBCE, SBCE findings, cross-sectional/radiological imaging both before and after SBCE, subsequent clinical outcomes. Iron deficiency anemia (IDA) was defined as per World Health Organisation guidelines: $\mathrm{Hb}<13 \mathrm{~g} / \mathrm{dL}$ in males or $<12 \mathrm{~g} / \mathrm{dL}$ in females, with evidence of iron deficiency (MCV $<80 \mathrm{fL}$ or ferritin $<12-15 \mu \mathrm{g} / \mathrm{L}$ ).

A literature review of the databases PubMed and Embase was also conducted for publications reporting the epidemiology of small bowel tumors. This was carried out via a focused search using the terms "small bowel tumors" and "small bowel malignancy" as keywords. Relevant studies were included if they reported the incidence of small bowel tumors in their cohorts.

\section{Results}

The total number of SBCE studies carried out between 2005 and

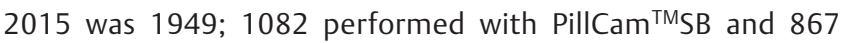
using MiroCam ${ }^{\mathrm{TM}}$. Small bowel malignancy was confirmed in 7 patients $(0.36 \% ; 5$ male/2 female). The median age was 50 years (range 34-67). There were 2 lymphomas, 2 gastrointestinal stromal tumors (GISTs), 2 duodenal adenocarcinomas and 1 jejunal metastasis from a sarcoma of the lung. In this subgroup, indications for CE were IDA (iron-deficiency anemia) $(n=5)$, unexplained diarrhoea $(n=1)$ and clinical suspicion of lymphoma $(n=1)$. The median time from first symptom to diagnosis in our patients was 12 months (range $2-18$ ). All patients with a small bowel malignancy had other investigations carried out prior to SBCE. Six of 7 had prior negative bidirectional gastrointestinal endoscopy. In 2 patients, SBCE was carried out immediately following negative UGIE and colonosco- py. One patient had a normal upper gastrointestinal endoscopy (UGIE) with no colonoscopy carried out. Other prior investigations before SBCE included: abdominal ultrasound scan (USS) $(n=2)$, computed tomography (CT) imaging of the chest, abdomen and/or pelvis $(n=4)$, small bowel barium follow-through $(n=1)$ and bone marrow aspirate $(n=1)$. The mean number of diagnostic procedures per patient, before CE, was 3. All prior diagnostic procedures were normal or nondiagnostic. Two of the 7 patients experienced asymptomatic capsule retention. Both these patients had duodenal adenocarcinomas; 1 required capsule removal by push enteroscopy and the other by UGIE.

All 7 patients had further investigations following CE. Six had a CT scan of their chest, abdomen, and pelvis carried out for staging. Two patients had push enteroscopy (PE), both of whom had a diagnosis of duodenal adenocarcinoma. One had double balloon enteroscopy (DBE), 2 had colonoscopy, 2 had UGIE; and there was 1 bone marrow aspiration. All patients had histological confirmation of malignancy. The SBCE findings (and subsequent investigations) led to a change in management in all our patients diagnosed with a small bowel malignancy. Three subjects had a small bowel resection. One patient with a GIST was also administered Imatinib following resection. Of the 2 individuals with a duodenal adenocarcinoma, 1 underwent a gastroenterostomy and 1 had an elective Whipple procedure. Four patients died within 1 year of their diagnosis, 2 of whom died after surgery and the other 2 before their planned surgery. Of the 3 surviving patients at the time of writing, 2 are being followed up by the oncology team and one by the gastroenterology team. Table 1 summarizes the findings.

\section{Discussion}

Introduction of wireless CE into clinical practice has radically changed diagnostic algorithms for small bowel pathology [ 9 , 11]. However, there is a growing body of evidence suggesting a high miss rate for sinister small bowel pathology using CE [12-15]. Radiological modalities, such as small bowel followthrough, enteroclysis and cross-sectional imaging ( $C T$ and magnetic resonance imaging), permit detection of lesions in the whole small bowel but provide inadequate detail of the bowel lumen and mucosa [16]. In our cohort, our patients underwent a similar number of investigations preceding SBCE compared to other studies (range 3.19-4.6) (See $>$ Table 2 for a comparison of previous studies on SB malignancy diagnosed by CE) $[17-43]$.

The reported rate of small bowel malignancy diagnosis by CE varies. Our cohort has a very low reported frequency of small bowel malignancy diagnosis at $0.36 \%$. Our results are consistent with other published studies on the detection of SB malignancies by CE, detailed in > Table 2. Notably, in a large multicenter study by Rondonotti et al with 5129 CEs from 29 centers in 10 European countries, the authors observed a significant inverse correlation between the frequency of tumors diagnosed and the number of CE examinations performed at a particular center [10]. In contrast, this single-center study originated at a tertiary hospital serving a large but relatively homogeneous 
- Table 1 Case-based demographics, clinical findings, investigations, management, and outcomes in 7 Patients with small bowel tumor diagnosis

\begin{tabular}{|c|c|c|c|c|c|c|c|c|}
\hline $\begin{array}{l}\text { Patient } \\
\text { Number }\end{array}$ & $\begin{array}{l}\text { Indica- } \\
\text { tion }\end{array}$ & $\begin{array}{l}\text { Time from } \\
\text { presenta- } \\
\text { tion to } \\
\text { diagnosis }\end{array}$ & $\begin{array}{l}\text { Diagnosis and } \\
\text { CE appearances }\end{array}$ & $\begin{array}{l}\text { Previous In- } \\
\text { vestigations }\end{array}$ & $\begin{array}{l}\text { Subsequent } \\
\text { Investiga- } \\
\text { tions }\end{array}$ & $\begin{array}{l}\text { Mode used to } \\
\text { confirm diag- } \\
\text { nosis and find- } \\
\text { ings }\end{array}$ & $\begin{array}{l}\text { Manage- } \\
\text { ment }\end{array}$ & Outcome \\
\hline 1 & IDA & 2 months & $\begin{array}{l}\text { Duodenal ade- } \\
\text { nocarcinoma: } \\
\text { Stricturing, } \\
\text { nodular mucosa, } \\
\text { CE retained }\end{array}$ & $\begin{array}{l}\text { Abdominal } \\
\text { USS, UGIE, } \\
\text { colonoscopy }\end{array}$ & $\begin{array}{l}\text { UGIE, PE, } \\
\text { CT CAP }\end{array}$ & $\begin{array}{l}\text { UGIE: } \\
\text { Tumor in duo- } \\
\text { denum and re- } \\
\text { tained CE }\end{array}$ & $\begin{array}{l}\text { Gastroenter- } \\
\text { ostomy and } \\
\text { palliative } \\
\text { care }\end{array}$ & Deceased \\
\hline 2 & IDA & 18 months & $\begin{array}{l}\text { GIST (right iliac } \\
\text { fossa): } \\
\text { Mucosal bulge }\end{array}$ & $\begin{array}{l}\text { CT, UGIE, } \\
\text { colonoscopy }\end{array}$ & CT CAP & $\begin{array}{l}\text { CT CAP: } \\
\text { Soft tissue no- } \\
\text { dules with flecks } \\
\text { of calcification }\end{array}$ & $\begin{array}{l}\text { Small bowel } \\
\text { resection }\end{array}$ & $\begin{array}{l}\text { Gl follow- } \\
\text { up }\end{array}$ \\
\hline 3 & IDA & 2 months & $\begin{array}{l}\text { Jejunal metas- } \\
\text { tasis from sarco- } \\
\text { ma of lung: } \\
\text { Multiple infiltra- } \\
\text { tive lesions }\end{array}$ & $\begin{array}{l}\text { CT chest, } \\
\text { BMA, UGIE }\end{array}$ & DBE, CT CAP & $\begin{array}{l}\text { DBE: } \\
\text { Area of intussus- } \\
\text { ception with } \\
\text { jejunal lesions }\end{array}$ & $\begin{array}{l}\text { Small bowel } \\
\text { resection } \\
\text { and pallia- } \\
\text { tive care }\end{array}$ & Deceased \\
\hline 4 & $\begin{array}{l}\text { Possible } \\
\text { lym- } \\
\text { phoma }\end{array}$ & 11 months & $\begin{array}{l}\text { Lymphoma } \\
\text { (diffuse): } \\
\text { Extensive pseu- } \\
\text { dopolyp forma- } \\
\text { tion at terminal } \\
\text { ileum }\end{array}$ & $\begin{array}{l}\text { Small bowel } \\
\text { follow } \\
\text { through, } \\
\text { UGIE, colo- } \\
\text { noscopy, CT } \\
\text { CAP }\end{array}$ & $\begin{array}{l}\text { Colonos- } \\
\text { copy }\end{array}$ & $\begin{array}{l}\text { Colonoscopy: } \\
\text { Extensive pseu- } \\
\text { dopolyp forma- } \\
\text { tion }\end{array}$ & $\begin{array}{l}\text { Planned } \\
\text { surgery }\end{array}$ & $\begin{array}{l}\text { Deceased } \\
\text { ( } 3 \text { months } \\
\text { after } \\
\text { diagnosis) }\end{array}$ \\
\hline 5 & IDA & 13 months & $\begin{array}{l}\text { Duodenal ade- } \\
\text { nocarcinoma: } \\
\text { Capacious } \\
\text { duodenum, ob- } \\
\text { structing lesion } \\
\text { with infiltrative } \\
\text { characteristics } \\
\text { in proximal jeju- } \\
\text { num, CE retained }\end{array}$ & $\begin{array}{l}\text { UGIE, colo- } \\
\text { noscopy, ab- } \\
\text { dominal USS, } \\
\text { CT }\end{array}$ & PE, CT CAP & $\begin{array}{l}\text { PE: } \\
\text { Tumor in duo- } \\
\text { denum and re- } \\
\text { tained CE }\end{array}$ & $\begin{array}{l}\text { Elective } \\
\text { Whipple } \\
\text { procedure }\end{array}$ & $\begin{array}{l}\text { Oncology } \\
\text { follow-up }\end{array}$ \\
\hline 6 & IDA & 16 months & $\begin{array}{l}\text { GIST (jejunal): } \\
\text { Mucosal bulge }\end{array}$ & $\begin{array}{l}\text { UGIE, colo- } \\
\text { noscopy }\end{array}$ & CT CAP & $\begin{array}{l}\text { CT CAP: } \\
\text { Area of mucosal } \\
\text { thickening and } \\
\text { inflammatory } \\
\text { changes at duo- } \\
\text { denal-jejunal } \\
\text { junction, reac- } \\
\text { tive lymph nodes }\end{array}$ & $\begin{array}{l}\text { Small bowel } \\
\text { resection } \\
\text { and imatinib }\end{array}$ & $\begin{array}{l}\text { Oncology } \\
\text { follow-up }\end{array}$ \\
\hline 7 & $\begin{array}{l}\text { Diar- } \\
\text { rhoea }\end{array}$ & unknown & $\begin{array}{l}\text { Lymphoma } \\
\text { (diffuse): } \\
\text { Infiltrative } \\
\text { appearances }\end{array}$ & $\begin{array}{l}\text { UGIE, colo- } \\
\text { noscopy }\end{array}$ & $\begin{array}{l}\text { UGIE, colo- } \\
\text { noscopy, CT } \\
\text { CAP, BMA }\end{array}$ & $\begin{array}{l}\text { UGIE: } \\
\text { Severe rugal } \\
\text { hyperplasia }\end{array}$ & $\begin{array}{l}\text { Planned } \\
\text { surgery }\end{array}$ & $\begin{array}{l}\text { Deceased } \\
\text { ( } 2 \text { months } \\
\text { after } \\
\text { diagnosis) }\end{array}$ \\
\hline
\end{tabular}

BMA, bone marrow aspiration; CE, capsule endoscopy; CT CAP, computed tomography of chest, abdomen and pelvis; IDA, iron deficiency anaemia; PE, push enteroscopy; UGIE, upper gastrointestinal endoscopy; USS, ultrasound scan

population from southeast Scotland. Furthermore, the lower incidence of small bowel malignancy found on CE in our group may also, to some extent, be an artifact of less stringent local CE referral policies due to the accessibility of the CE service. This is reflected by the overall high proportion of normal CEs (971/1949; 49.8\%). A significant 51.0\% (994/1949) of our referrals were for obscure gastrointestinal bleeding and $41.3 \%$ (805/1949) specifically for IDA; the low diagnostic yield is in line with that of previous published studies and systematic reviews on CE in patients with IDA [44].
With 1949 CE reports, our study represents one of the largest single-center studies to date reporting on small bowel malignancies. Fig. 1 shows the forest plot for the rate of small bowel tumors detected by $\mathrm{CE}$ in the studies from $>$ Table 2: using the random effects model (DerSimonian-Laird) due to high study heterogeneity, the overall proportion of small bowel tumors diagnosed on CE was 0.05 ( $95 \% \mathrm{Cl} 0.04-0.06)$, i. e. $5 \%$. When only malignant tumors (if specified by the respective studies) were taken into account, the rate of diagnosis via CE was 0.03 (95\% Cl $0.02-0.04)$, or $3 \%$, > Fig. 2 . 


\begin{tabular}{|c|c|c|c|c|c|c|c|c|c|c|c|c|c|c|c|c|}
\hline $\begin{array}{l}\dot{\tilde{u}} \\
\stackrel{\tilde{u}}{\tilde{\Sigma}}\end{array}$ & & 1 & - & & & $m$ & 1 & I & & 1 & 1 & - & $\stackrel{\simeq}{\sim}$ & $\nabla$ & 1 & \\
\hline 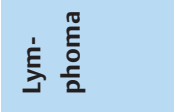 & & 1 & I & & & - & 乞n & $m$ & & 1 & $m$ & $\sigma$ & $\simeq$ & $m$ & - & \\
\hline$\frac{5}{v}$ & & 1 & 1 & & & $m$ & I & $\sim$ & & $\sim$ & 0 & $m$ & $\stackrel{\infty}{m}$ & $\sim$ & - & \\
\hline 离 & & 1 & I & & & 0 & $\circ$ & - & & 1 & - & $\approx$ & $\approx$ & $m$ & 1 & \\
\hline 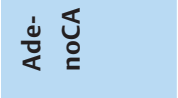 & 1 & - & 1 & 1 & 1 & in & $a$ & $\nabla$ & 1 & $\nabla$ & $m$ & $\stackrel{\infty}{\sim}$ & $\stackrel{m}{\sim}$ & - & - & \\
\hline 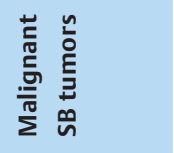 & $\tilde{z}$ & - & - & $\tilde{z}$ & $\tilde{z}$ & $\stackrel{\infty}{\Gamma}$ & $\stackrel{\stackrel{n}{N}}{ }$ & $\mp$ & $\tilde{z}$ & $\infty$ & $\stackrel{m}{\sim}$ & กn & $\stackrel{\infty}{\circ}$ & $\stackrel{m}{\sim}$ & $\nabla$ & $\tilde{z}$ \\
\hline 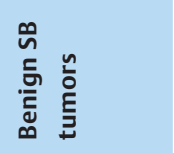 & $\tilde{z}$ & $\sigma$ & 0 & $\tilde{z}$ & $\tilde{z}$ & $a$ & $\stackrel{\circ}{\leftarrow}$ & 0 & $\tilde{z}$ & $\sim$ & $\sim$ & $\stackrel{\stackrel{n}{m}}{m}$ & 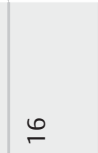 & 0 & 0 & $\tilde{z}$ \\
\hline 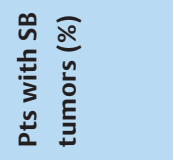 & $\begin{array}{l}\widehat{0} \\
\stackrel{0}{0} \\
\underline{0}\end{array}$ & 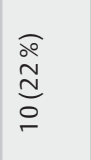 & 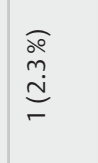 & $\begin{array}{l}\text { ò } \\
\stackrel{m}{n} \\
\stackrel{m}{m}\end{array}$ & 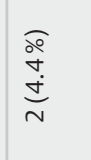 & 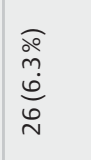 & $\begin{array}{l}\text { बo } \\
\stackrel{0}{0} \\
\stackrel{\omega}{n} \\
m\end{array}$ & $\begin{array}{l}\text { od } \\
\stackrel{d}{d} \\
\check{c} \\
\tau\end{array}$ & $\begin{array}{l}\stackrel{\text { o }}{n} \\
\stackrel{n}{n}\end{array}$ & $\begin{array}{l}\overline{\text { o }} \\
\stackrel{m}{a}\end{array}$ & 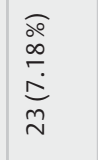 & $\infty$ & 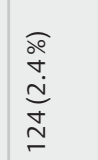 & $\begin{array}{l}\text { ò } \\
\dot{v} \\
\stackrel{m}{m} \\
m\end{array}$ & $\begin{array}{l}\widehat{d} \\
\stackrel{d}{\sigma}\end{array}$ & $\begin{array}{l}\text { ळo } \\
\infty \\
\text { ம் } \\
\sigma\end{array}$ \\
\hline 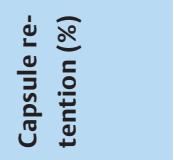 & 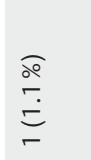 & $\tilde{z}$ & 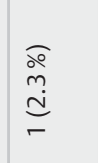 & 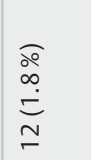 & $\tilde{z}$ & $\begin{array}{l}\bar{d} \\
\hat{0} \\
\stackrel{0}{m}\end{array}$ & $\tilde{z}$ & $\tilde{z}$ & $\begin{array}{l}\text { ১o } \\
\infty \\
\stackrel{0}{0} \\
\text { N }\end{array}$ & 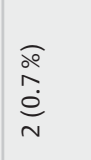 & $\tilde{z}$ & $\tilde{z}$ & $\begin{array}{l}\frac{\mathfrak{o}}{\grave{c}} \\
\stackrel{0}{a} \\
\end{array}$ & $\begin{array}{l}\bar{\alpha} \\
\infty \\
0 \\
\text { m }\end{array}$ & $\tilde{z}$ & $\tilde{z}$ \\
\hline 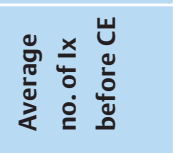 & $m$ & $\tilde{z}$ & $\bar{\sigma}$ & $\tilde{z}$ & $\tilde{z}$ & $\begin{array}{l}\stackrel{q}{m} \\
\text { m. }\end{array}$ & 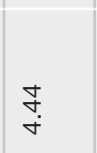 & $\stackrel{\varphi}{\dot{m}}$ & $\tilde{z}$ & $\begin{array}{l}\stackrel{J}{\Delta} \\
\stackrel{0}{0}\end{array}$ & $\tilde{z}$ & $\begin{array}{l}\qquad \\
\dot{\sigma}\end{array}$ & $\begin{array}{l}\tilde{\infty} \\
\sim \\
\sim\end{array}$ & in & $m$ & $\tilde{z}$ \\
\hline 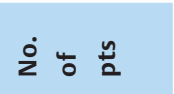 & $\infty$ & g & $\stackrel{m}{\forall}$ & గู & 年 & $\stackrel{0}{\sigma}$ & 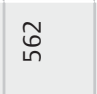 & $\stackrel{m}{f}$ & 怘 & 品 & $\stackrel{\curvearrowright}{\text { ల }}$ & $\tilde{z}$ & $\underset{\text { in }}{\stackrel{9}{N}}$ & $\stackrel{\circ}{\infty}$ & $\bullet$ & $\stackrel{\stackrel{\llcorner}{\llcorner}}{\llcorner}$ \\
\hline 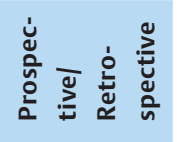 & 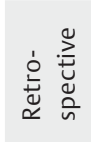 & 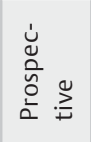 & 完总 & 它 & 客 & 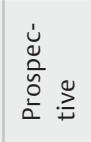 & 它 & 完密 & 它 & 它总 & 它总 & 它总 & : & 竞 & 蓠总 & 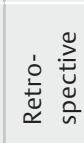 \\
\hline 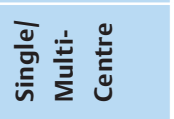 & $\begin{array}{l}\frac{\sigma}{\bar{T}} \\
\stackrel{\bar{n}}{n}\end{array}$ & $\begin{array}{l}\frac{0}{\pi} \\
\stackrel{5}{n}\end{array}$ & $\begin{array}{l}\frac{0}{\bar{T}} \\
\stackrel{5}{5}\end{array}$ & $\begin{array}{l}\frac{\Delta}{\bar{N}} \\
\dot{\bar{n}}\end{array}$ & $\begin{array}{l}\frac{\varrho}{\bar{T}} \\
\stackrel{5}{n}\end{array}$ & $\frac{\bar{E}}{\sum}$ & $\begin{array}{l}\frac{0}{\bar{T}} \\
\stackrel{5}{\bar{n}}\end{array}$ & $\frac{\bar{E}}{\bar{J}}$ & $\begin{array}{l}\frac{0}{\bar{T}} \\
\stackrel{\bar{n}}{n}\end{array}$ & $\begin{array}{l}\frac{\varrho}{\bar{T}} \\
\stackrel{\Xi}{\bar{n}}\end{array}$ & $\begin{array}{l}\frac{0}{\tilde{G}} \\
\stackrel{5}{n}\end{array}$ & $\frac{\bar{I}}{\bar{J}}$ & $\frac{\bar{T}}{\bar{J}}$ & $\begin{array}{l}\frac{0}{\tilde{T}} \\
\stackrel{5}{5}\end{array}$ & $\begin{array}{l}\frac{\varrho}{\bar{T}} \\
\stackrel{\bar{\sigma}}{n}\end{array}$ & $\begin{array}{l}\frac{0}{\pi} \\
\stackrel{\bar{n}}{n}\end{array}$ \\
\hline ذ̇ & $\begin{array}{l}\text { 売 } \\
\hat{n}\end{array}$ & $\begin{array}{l}\stackrel{\Xi}{\check{L}} \\
\text { 趈 }\end{array}$ & ș & 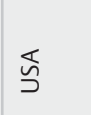 & 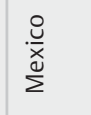 & 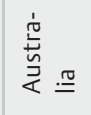 & 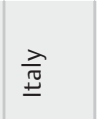 & $\frac{E}{\frac{E}{5}}$ & 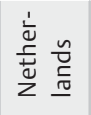 & 气̀ & 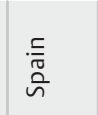 & 气 & 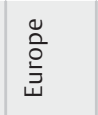 & 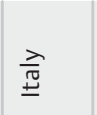 & $\begin{array}{l}\text { 仓े } \\
\text { 音 }\end{array}$ & 胥 \\
\hline 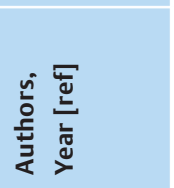 & 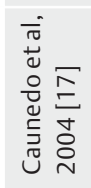 & 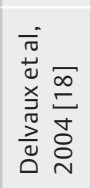 & 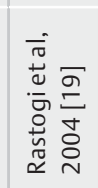 & 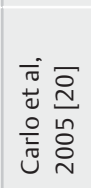 & 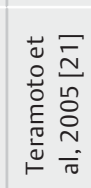 & 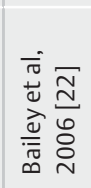 & 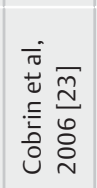 & 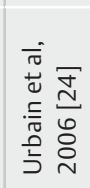 & 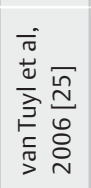 & 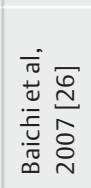 & 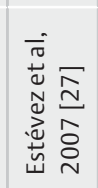 & 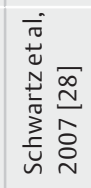 & 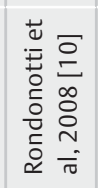 & 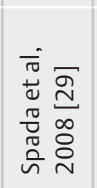 & 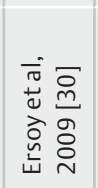 & 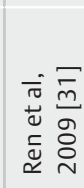 \\
\hline
\end{tabular}




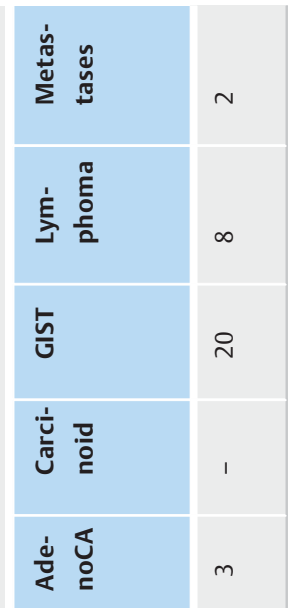

\section{II}
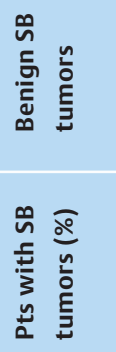

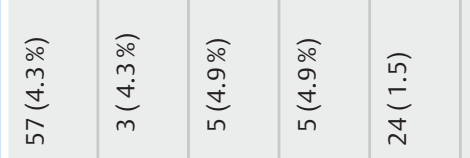

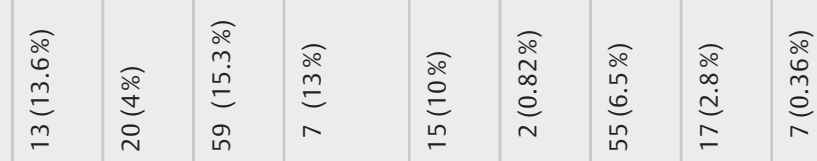

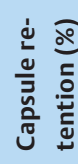

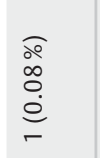

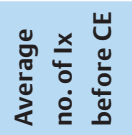

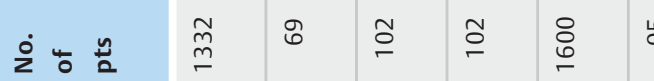

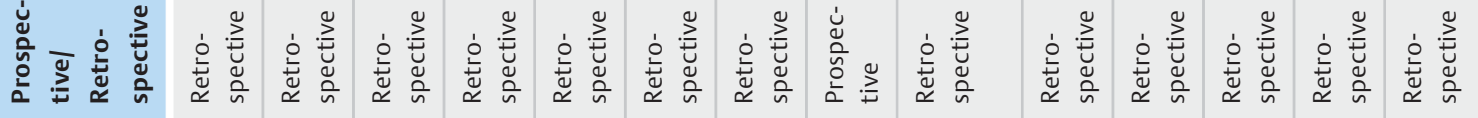

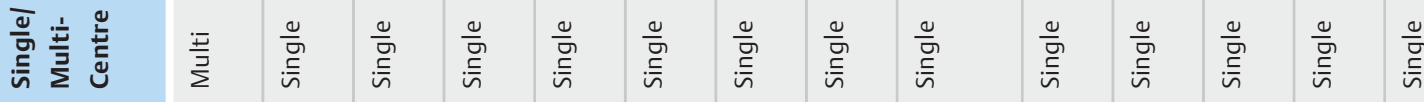

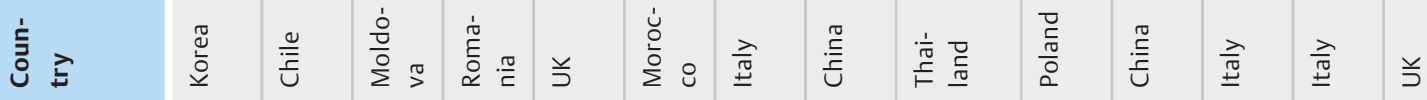

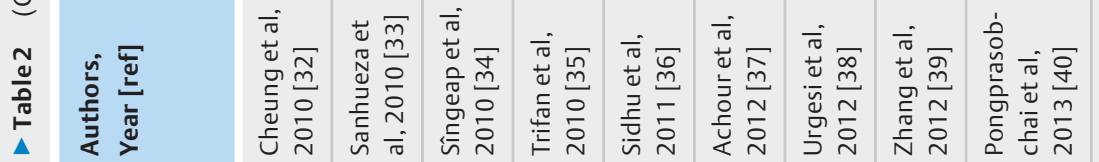

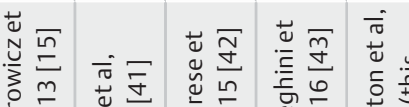

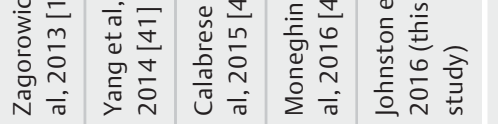


Caunedo et al 2004

Delvaux et al 2004

Rastogi et al 2004

Carlo et al 2005

Teramoto et al 2005

Bailey et al 2006

Cobrin et al 2006

Urbain et al 2006

van Tuyl et al 2006

Baichi et al 2007

Estévez et al 2007

Rondonotti et al 2008

Spada et al 2008

Ersoy et al 2009

Ren et al 2009

Cheung et al 2010

Sanhueza et al 2010

Sîngeap et al 2010

Trifan et al 2010

Sidhu et al 2011

Achour et al 2012

Urgesi et al 2012

Zhang et al 2012

Pongprasopchai et al 2013

Zagorowicz et al 2013

Yang et al 2014

Calabrese et al 2015

Moneghini et al 2016

Johnston et al 2016

combined

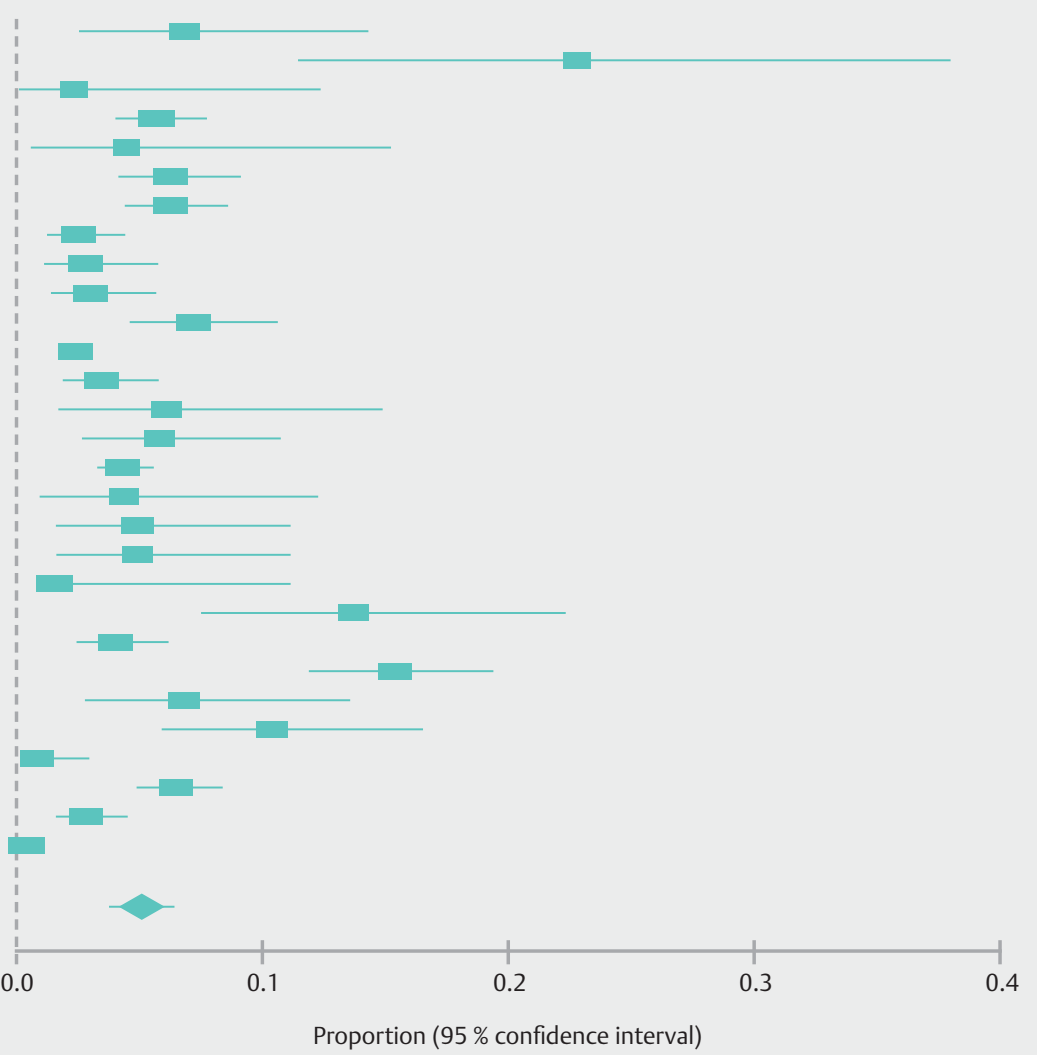

Proportion (95\% confidence interval)

Cochran $Q=356.301055(\mathrm{df}=28) P<0.0001$

Moment-based estimate of between studies variance $=\mathbf{0 . 0 2 2 2 2 3}$

$\mathrm{I}^{2}$ (inconsistency) $=\mathbf{9 2 . 1} \%(95 \% \mathrm{Cl}=\mathbf{9 0 . 2} \%$ to $93.5 \%)$
$0.07(0.03,0.14)$

$0.23(0.11,0.38)$

$0.02(0.00059,0.12)$

$0.06(0.04,0.08)$

$0.04(0.0054,0.15)$

$0.06(0.04,0.09)$

$0.06(0.04,0.09)$

$0.02(0.01,0.04)$

$0.03(0.01,0.06)$

$0.03(0.01,0.06)$

$0.07(0.05,0.11)$

$0.02(0.02,0.03)$

$0.03(0.02,0.06)$

$0.06(0.02,0.15)$

$0.06(0.03,0.11)$

$0.04(0.03,0.06)$

$0.04(0.0091,0.12)$

$0.05(0.02,0.11)$

$0.05(0.02,0.11)$

$0.02(0.0096,0.02)$

$0.14(0.07,0.22)$

$0.04(0.02,0.06)$

$0.15(0.12,0.19)$

$0.07(0.03,0.14)$

$0.10(0.06,0.16)$

$0.0082(0.001,0.03)$

$0.06(0.05,0.08)$

$0.03(0.02,0.04)$

$0.0036(0.0014,0.0074)$

$0.05(0.04,0.06)$

- Fig. 1 Forest plot showing pooled rate of diagnosis of small bowel tumors by CE.

The most common indication for CE in our study was IDA (71.4\%). This matches the published reports on small bowel cancers diagnosed through CE, where IDA accounted for $60 \%$ to $100 \%$ of indications. In our group, malignancy was diagnosed more frequently in younger patients $(\leq 55 \mathrm{y})$ with IDA ( 3 of 312 CE cases, $0.96 \%$ ) compared with those older than age 55 years ( 2 of 682 CE cases, $0.29 \%$ ). Our findings highlight the importance of keeping small bowel malignancy on the differential diagnosis in younger patients with IDA [44, 45].

Early detection of small bowel malignancy has a positive impact on survival. Overall, the prognosis of small bowel tumors remains poor [46-48] and a diagnostic delay of up to 1.5 years has been estimated for malignant small bowel tumors. Features of small bowel tumors contributing to delayed diagnosis include their slow, extraluminal growth and lack of specific symptoms [10].

The major limitations of this observational study are its retrospective design and the fact that information on follow-up was only available for a limited number of patients in our cohort. This was due to the large catchment area of our tertiary referral center, which meant that a significant proportion of patients were returned to the care of their referring centers fol- lowing CE. However, it still represents one of the largest studies to date on small bowel cancers diagnosed by CE.

\section{Conclusion}

In summary, in our tertiary care center, the rate of diagnosis of small bowel malignancies via CE was $0.36 \%$. This was a low diagnostic yield in comparison to other studies, but possibly a truer figure given our large sample size from a single center. Younger patients referred for CE with IDA were more likely to be diagnosed with small bowel cancer. The mortality rate for patients diagnosed this way was high, with a 5-year survival rate of only $42.9 \%$. The potential diagnostic superiority of CE over other small bowel investigations and the resultant change in management plans suggest that CE is an invaluable third-line diagnostic procedure following negative bidirectional gastrointestinal endoscopy when other imaging modalities have failed. 
Delvaux et al 2004

Rastogi et al 2004

Bailey et al 2006

Cobrin et al 2006

Urbain et al 2006

Baichi et al 2007

Estévez et al 2007

Rondonotti et al 2008

Spada et al 2008

Ersoy et al 2009

Cheung et al 2010

Trifan et al 2010

Sidhu et al 2011

Achour et al 2012

Zhang et al 2012

Zagorowicz et al 2013

Yang et al 2014

Calabrese et al 2015

Moneghini et al 2016

Johnston et al 2016

combined

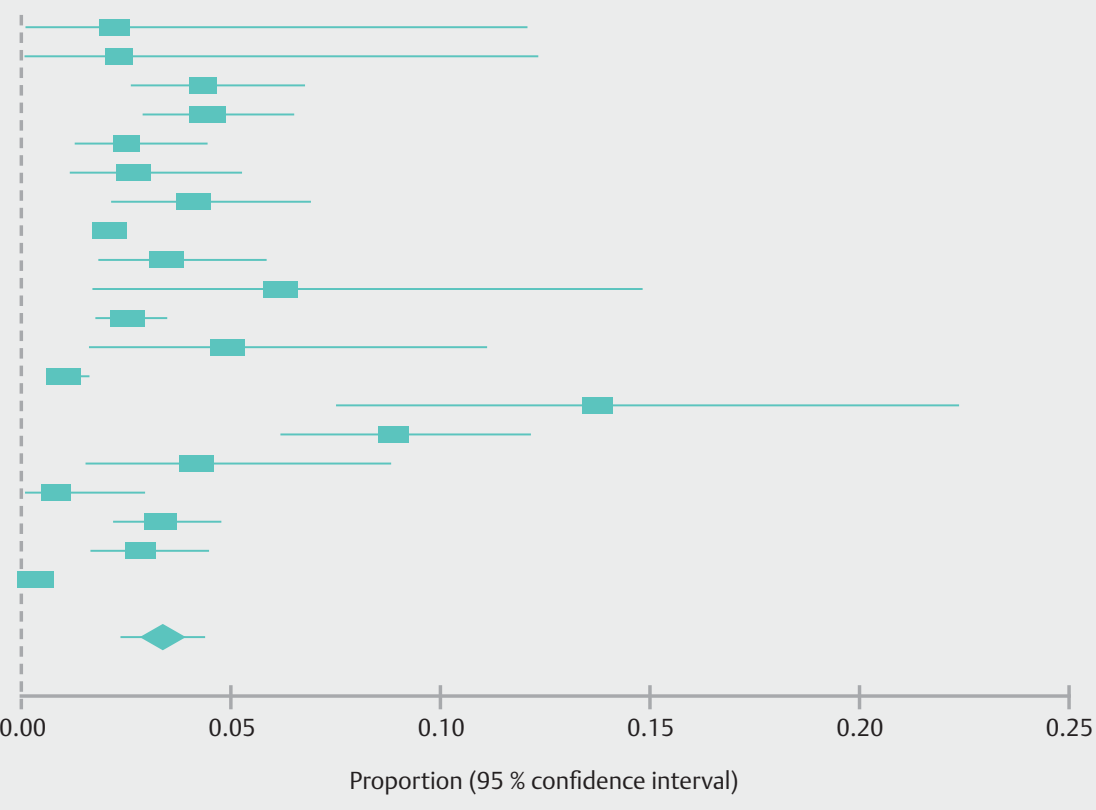

$0.02(0.00058,0.12)$

$0.02(0.00059,0.12)$

$0.04(0.03,0.07)$

$0.04(0.03,0.06)$

$0.02(0.01,0.04)$

$0.03(0.01,0.05)$

$0.04(0.02,0.07)$

$0.02(0.02,0.03)$

$0.03(0.02,0.06)$

$0.06(0.02,0.15)$

$0.02(0.02,0.03)$

$0.05(0.02,0.11)$

$0.01(0.0057,0.02)$

$0.14(0.07,0.22)$

$0.09(0.06,0.12)$

$0.04(0.02,0.09)$

$0.0082(0.001,0.03)$

$0.03(0.02,0.05)$

$0.03(0.02,0.04)$

$0.0036(0.0014,0.0074)$

$0.03(0.02,0.04)$

Cochran $Q=172.424664(\mathrm{df}=19) P<0.0001$

Moment-based estimate of between studies variance $=\mathbf{0 . 0 1 2 2 0 8}$

$\mathrm{I}^{2}$ (inconsistency) $=89 \%(95 \% \mathrm{Cl}=\mathbf{8 4 . 8} \%$ to $91.6 \%)$

- Fig. 2 Forest plot showing pooled rate of diagnosis of malignant small bowel tumors by CE.

Competing interests

None

\section{References}

[1] Schottenfeld D, Beebe-Dimmer JL, Vigneau FD. The Epidemiology and Pathogenesis of Neoplasia in the Small Intestine. Ann Epidemiol 2009; 19: $58-69$

[2] Pan SY, Morrison H. Epidemiology of cancer of the small intestine. World J Gastrointest Oncol 2011; 3: 33 -42

[3] De Franchis R, Rondonotti E, Abbiati C et al. Small bowel malignancy. Gastrointest Endosc Clin N Am 2004; 14: 139-148

[4] Talamonti M, Goetz L, Rao S et al. Primary cancers of the small bowel: analysis of prognostic factors and results of surgical management. Arch Surg 2002; 137: 564 - 570

[5] Pennazio M, Rondonotti E, de Franchis R. Capsule endoscopy in neoplastic diseases. World J Gastroenterol 2008; 14: 5245 - 5253

[6] Dabaja BS, Suki D, Pro B et al. Adenocarcinoma of the Small Bowel: Presentation, Prognostic Factor, and Outcome of 217 Patients. 2004; 101: $518-526$

[7] Vagholkar K, Mathew T. Adenocarcinoma of the small bowel: a surgical dilemma. Saudi J Gastroenterol 2009; 15: 264-267

[8] Rangiah DS, Cox M, Richardson M et al. Small bowel tumors: A 10 year experience in four Sydney teaching hospitals. ANZ J Surg 2004; 74: $788-792$

[9] Pennazio M, Spada C, Eliakim R et al. Small-bowel capsule endoscopy and device-assisted enteroscopy for diagnosis and treatment of small-bowel disorders: European Society of Gastrointestinal Endoscopy (ESGE) Clinical Guideline. Endoscopy 2015; 47: 352 - 376
[10] Rondonotti E, Pennazio M, Toth E et al. Small-bowel neoplasms in patients undergoing video capsule endoscopy: A multicenter European study. Endoscopy 2008; 40: 488-495

[11] Wang A, Banerjee S, Barth B et al. TECHNOLOGY STATUS EVALUATION REPORT: Wireless capsule endoscopy. Gastrointest Endosc 2013; 63: $539-545$

[12] Rondonotti E, Herrerias JM, Pennazio M et al. Complications, limitations, and failures of capsule endoscopy: A review of 733 cases. Gastrointest Endosc 2005; 62: 712 - 716

[13] Postgate A, Despott E, Burling D et al. Significant small-bowel lesions detected by alternative diagnostic modalities after negative capsule endoscopy. Gastrointest Endosc 2008; 68: 1209-1214

[14] Chong AKH, Chin BWK, Meredith CG. Clinically significant small-bowel pathology identified by double-balloon enteroscopy but missed by capsule endoscopy. Gastrointest Endosc 2006; 64: 445 - 449

[15] Zagorowicz ES, Pietrzak AM, Wronska E et al. Small bowel tumors detected and missed during capsule endoscopy: Single center experience. World J Gastroenterol 2013; 19: 9043 - 9048

[16] Saperas E, Dot J, Videla S et al. Capsule endoscopy versus computed tomographic or standard angiography for the diagnosis of obscure gastrointestinal bleeding. Am J Gastroenterol 2007; 102: 731 - 737

[17] Caunedo A, Rodríguez-Téllez M, García-Montes JM et al. Usefulness of capsule endoscopy in patients with suspected small bowel disease. Rev Esp Enferm Dig 2004; 96: $10-21$

[18] Delvaux M, Fassler I, Gay G. Clinical Usefulness of the Endoscopic Video Capsule as the Initial Intestinal Investigation in Patients with Obscure Digestive Bleeding: Validation of a Diagnostic Strategy Based on the Patient Outcome after 12 Months. Endoscopy 2004; 36: $1067-1073$

[19] Rastogi A, Schoen RE, Slivka A. Diagnostic yield and clinical outcomes of capsule endoscopy. Gastrointest Endosc 2004; 60: 959 - 964 
[20] Carlo JT, DeMarco D, Smith BA et al. The utility of capsule endoscopy and its role for diagnosing pathology in the gastrointestinal tract. Am J Surg 2005; 190: $886-890$

[21] Teramoto Matsubara O, Zamarripa Dorsey F, López Acosta ME. [Capsule endoscopy: The evolution in the diagnosis of small bowel diseases]. Rev Gastroenterol México 2005; 70: 138 - 142

[22] Bailey AA, Debinski HS, Appleyard MN et al. Diagnosis and outcome of small bowel tumors found by capsule endoscopy: A three-center Australian experience. Am J Gastroenterol 2006; 101: 2237-2243

[23] Cobrin GM, Pittman RH, Lewis BS. Increased diagnostic yield of small bowel tumors with capsule endoscopy. Cancer 2006; 107: 22 - 27

[24] Urbain D, De Looze D, Demedts I et al. Video capsule endoscopy in small-bowel malignancy: A multicenter Belgian study. Endoscopy 2006; 38: $408-411$

[25] Van Tuyl SAC, Van Noorden JT, Kuipers EJ et al. Results of videocapsule endoscopy in 250 patients with suspected small bowel pathology. Dig Dis Sci 2006; 51: 900 - 905

[26] Baichi MM, Arifuddin RM, Mantry PS. Small-bowel masses found and missed on capsule endoscopy for obscure bleeding. Scand J Gastroenterol 2007; 42: 1127 - 1132

[27] Estevez E, Gonzalez-Conde B, Vazquez-Iglesias JL et al. Incidence of tumoral pathology according to study using capsule endoscopy for patients with obscure gastrointestinal bleeding. Surg Endosc Other Interv Tech 2007; 21: $1776-1780$

[28] Schwartz GD, Barkin JS. Small-bowel tumors detected by wireless capsule endoscopy. Dig Dis Sci 2007; 52: 1026 - 1030

[29] Spada C, Riccioni ME, Familiari P et al. Video capsule endoscopy in small-bowel tumors: a single center experience. Scand J Gastroenterol 2008; 43: 497-505

[30] Ersoy O, Harmanci O, Aydinli M et al. Capability of capsule endoscopy in detecting small bowel ulcers. Dig Dis Sci 2009; 54: 136-141

[31] Ren M, Yuan G, Zhu S et al. [Evaluation of capsule endoscopy in the diagnosis of small bowel disease]. Zhonghua Wei Chang Wai Ke Za Zhi 2009; 12: $163-166$

[32] Cheung DY, Lee IS, Chang DK et al. Capsule endoscopy in small bowel tumors: A multicenter Korean study. J Gastroenterol Hepatol 2010; 25: $1079-1086$

[33] Sanhueza Bravo E, Ibáñez P, Araya R et al. [Experience with capsule endoscopy diagnostic tool for the small intestine]. Rev Médica Chile 2010; 138: 303-308

[34] Sîngeap A-M, Trifan A, Cojocariu C et al. [Capsule endoscopy role in diagnosis of small bowel tumors]. Rev Medico-Chirurgicală a Soc Medici Şi Nat Din laşi 2010; 114: 988 - 992
[35] Trifan A, Singeap A-M, Cojocariu C et al. Small bowel tumors in patients undergoing capsule endoscopy: a single center experience. J Gastrointestin Liver Dis 2010; 19: 21 - 25

[36] Sidhu R, McAlindon ME. The Use of Capsule Endoscopy for the Investigation of Small Bowel Tumors: Experience from a United Kingdom Single Center. Dig Dis Sci 2011; 56: 2763

[37] Achour J, Serraj I, Amrani L et al. Small bowel tumors: What is the contribution of video capsule endoscopy? Clin Res Hepatol Gastroenterol 2012; 36: $222-226$

[38] Urgesi R, Riccioni ME, Bizzotto A et al. Increased diagnostic yield of small bowel tumors with PillCam: The role of capsule endoscopy in the diagnosis and treatment of gastrointestinal stromal tumors (GISTs). Italian single-center experience. Tumori 2012; 98: 357-363

[39] Zhang B, Chen C, Li Y. Capsule endoscopy examination identifies different leading causes of obscure gastrointestinal bleeding in patients of different ages. Turk J Gastroenterol 2012; 23: 220-225

[40] Pongprasobchai S, Chitsaeng S, Tanwandee T et al. Yield, etiologies and outcomes of capsule endoscopy in Thai patients with obscure gastrointestinal bleeding. World J Gastrointest Endosc 2013; 5: $122-127$

[41] Yang L, Chen Y, Zhang B et al. Increased diagnostic yield of capsule endoscopy in patients with chronic abdominal pain. PLoS One 2014; 9: e87396

[42] Calabrese C, Gionchetti P, Calafiore A et al. Sporadic small bowel tumors detected by capsule endoscopy in patients with occult gastrointestinal bleeding. Intern Emerg Med 2015; 10: 781 - 785

[43] Moneghini D, Missale G, Minelli L et al. P.18.1 SMALL BOWEL TUMORS IN PATIENTS UNDERGOING CAPSULE ENDOSCOPY: A SINGLE CENTER EXPERIENCE. Dig Liver Dis 2016; 48: e215 - e216

[44] Yung DE, Koulaouzidis A, Avni T etal. Clinical outcomes of negative small-bowel capsule endoscopy for small-bowelbleeding: a systematic review and meta-analysis. Gastrointest Endosc 2017; 85: 305-317

[45] Koulaouzidis A, Yung DE, Lam JHP et al. The use of small-bowel capsule endoscopy in iron-deficiency anemia alone; be aware of the young anemic patient. Scand J Gastroenterol 2012; 47: $1094-1100$

[46] Sidhu PS, McAlindon ME, Drew K et al. The Utility of Capsule Endoscopy in Patients under 50 Years of Age with Recurrent Iron Deficiency Anemia: Is the Juice Worth the Squeeze? Gastroenterol Res Pract 2015: 948574

[47] Shack LG, Wood HE, Kang JY et al. Small intestinal cancer in England \& Wales and Scotland: time trends in incidence, mortality and survival. Aliment Pharmacol Ther 2006; 23: 1297-1306

[48] Modlin IM, Lye KD, Kidd M. A 5-decade analysis of 13,715 carcinoid tumors. Cancer 2003; 97: 934-959 